\title{
Conversation au chevet de Wilder Penfield
}

$\mathrm{D}$ u $1^{\text {er }}$ juillet 1975 au 30 juin 1976, j'ai fait mon internat en médecine interne à l'Hôpital Royal Victoria de Montréal, au Québec, après avoir terminé mes études en médecine à l'Université Emory.

À cette époque là, les internes se voyaient encore attribuer une salle de garde individuelle. Mon rituel avant la tournée du matin : un beigne à l'érable et un jus d'orange que je prenais au café de l'hôpital, tout en dévorant The Star, The Gazette et La Presse. C'était la fièvre olympique. Les tensions séparatistes étaient toujours palpables et René Lévesque, chef charismatique du Parti Québécois, un parti séparatiste, faisait constamment la manchette, une cigarette perpétuellement à ses lèvres.

J'étais fasciné par le célèbre Institut neurologique de Montréal, où j'avais déjà assisté à une intervention chirurgicale, la suivant au moyen de jumelles de spectacle à partir d'une aire d'observation fermée qui surplombait la salle d'opération.

Je suis récemment tombé sur six pages de la taille d'une ordonnance médicale contenant des notes manuscrites que j'avais prises lors d'une conversation d'une heure avec le fondateur de l'Institut, le $\mathrm{D}^{\mathrm{r}}$ Wilder Penfield, au cours de la matinée du 3 avril 1976. Il était alors hospitalisé au Pavillon Ross du Royal Vic. Comme j'espérais le rencontrer depuis le début de ma formation, après avoir terminé ma garde de nuit, j'étais passé par ma chambre afin de trouver le courage de me présenter et de prendre mon exemplaire de son ouvrage The Torch, roman sur la lutte livrée par Hippocrate pour jeter les assises de la médecine.

Par ce beau samedi matin ensoleillé, le $\mathrm{D}^{\mathrm{r}}$ Penfield, alors âgé de 85 ans, était seul dans sa chambre. Il avait l'air fatigué et était pâle, mais serein. Lorsque j'ai frappé à la porte ouverte, il m'a gentiment invité à venir m'assoir à son chevet, en ajoutant «Je suis un cas intéressant, vous savez. Myopathie inflammatoire, de type myosite à inclu-

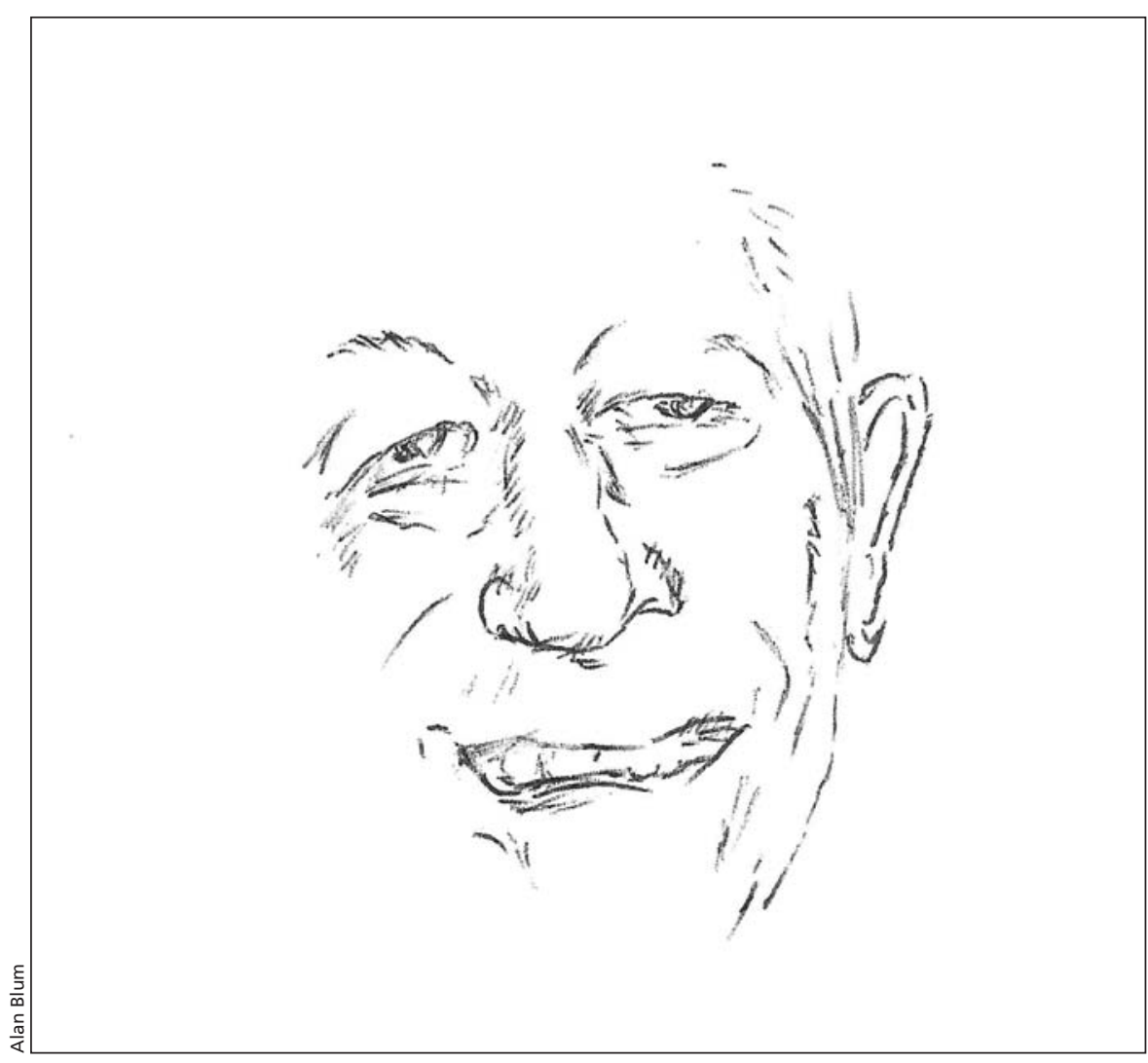

" Celui qui écrit avec son imagination sait ce qui est vrai ", a déclaré Wilder Penfield (cidessus) à l'auteur et artiste, Alan Blum.

sions. Vous avez lu mon dossier? Fichue myosite. Faiblesse. Je ne peux rien faire. Il y a aussi ce fichu cancer. »

Je lui ai dit que j'avais rencontré un de ses anciens patients, préposé à l'Hôpital des anciens combattants, qui avait été atteint d'une encéphalite virale 30 ans plus tôt et qu'il avait opéré.

« Je ne m'attends pas à lui avoir fait grand bien », a-t-il ajouté sur un ton railleur.

Il a rayonné de joie lorsque je lui ai montré mon exemplaire de poche de son livre The Torch. " J'ai eu tellement de plaisir à l'écrire, a-t-il dit. C'est vraiment la bonne façon, celle du romancier. Celui qui écrit avec son imagination sait ce qui est vrai. L'historien ne peut faire cela lorsqu'il dresse une liste des événements du passé. »

Ses propos étaient plutôt ironiques, car me voici 35 ans plus tard en train d'essayer de recréer notre conversation à partir de souvenirs personnels et de ces quelques notes conservées pendant toutes ces années. Cette rencontre revécue me laisse entrevoir ce qu' affirmait le $\mathrm{D}^{\mathrm{r}}$ Penfield : même si les mots ne sont pas exacts, les sensations évoquées par la rencontre sont vraies.

Le $\mathrm{D}^{\mathrm{r}}$ Penfield a continué, de me parler de son livre. « Lorsque je travaillais à l'Institute for Advanced Studies [à Princeton], les secrétaires de [J. Robert] Oppenheimer avaient dû se charger de taper le manuscrit. Oppenheimer avait vu ce dernier et avait déclaré "Je viens tout juste de voir le mot 'je' utilisé sur ma machine à écrire". Il était physicien, vous voyez, et n'avait jamais parlé à la première personne. »

Selon mes notes, le $\mathrm{D}^{\mathrm{r}}$ Penfield a dit : «Il n'y a pas beaucoup de mentions d'Hippocrate dans Osler. Du 


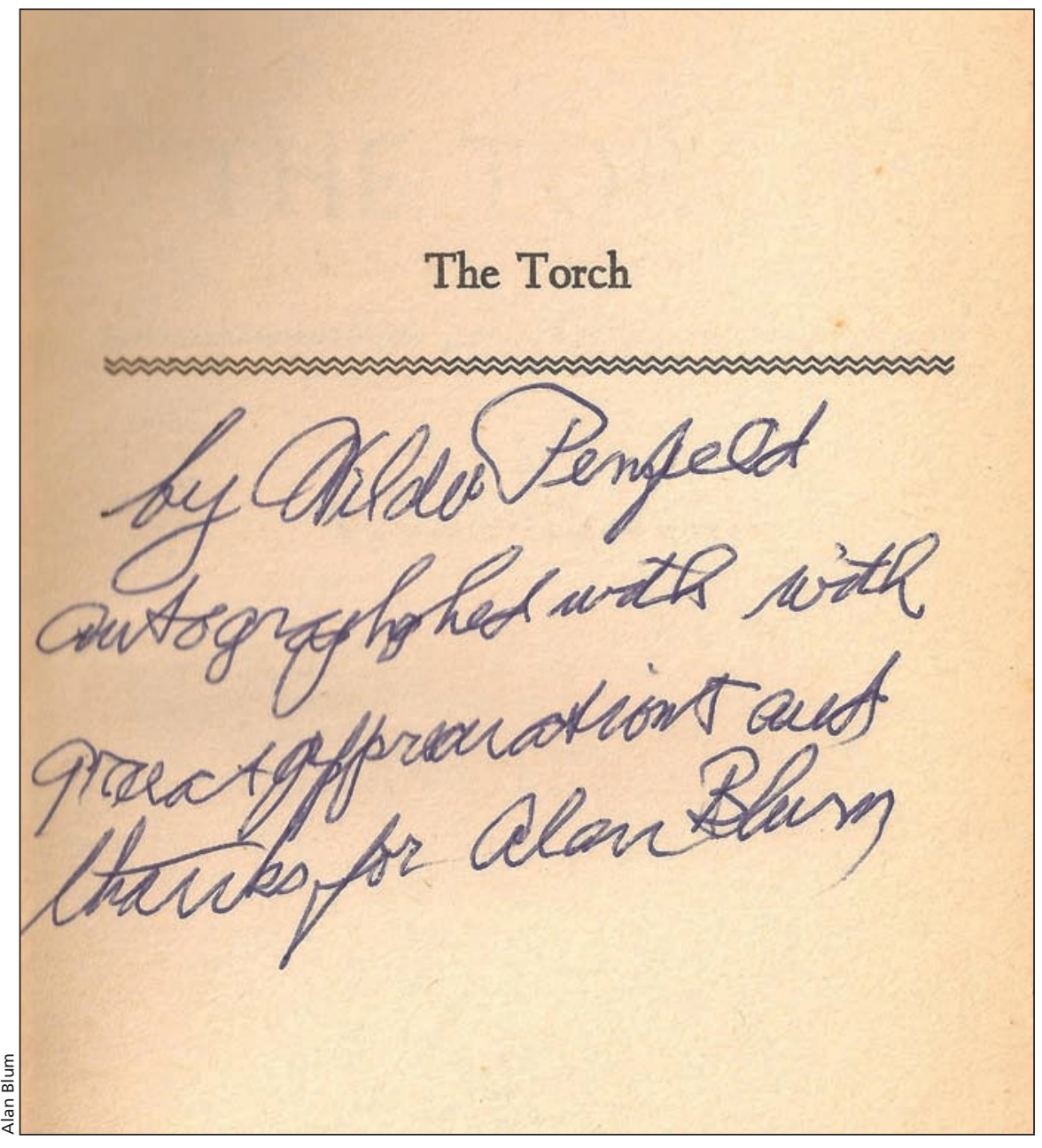

Le $D^{r}$ Blum a rendu visite au $D^{r}$ Penfield ce jour-là pour lui demander d'autographier son exemplaire du livre The Torch (ci-dessus).

moins pas de sa personne. Mais tout est là. » Je ne peux me souvenir du contexte exact, mis à part qu'il a fait allusion à The Torch, mais $\mathrm{D}^{\mathrm{r}}$ Penfield vénérait Sir William Osler, qui avait fait de lui un véritable membre de sa famille lorsque le $\mathrm{D}^{\mathrm{r}}$ Penfield avait été boursier Rhodes à Oxford et Sir Osler, professeur Regius de médecine.

Autre commentaire hors contexte : «L'assassinat de Socrate était en quelque sorte une grève. » C'était peutêtre une allusion à l'agitation qui régnait dans le milieu de travail hospitalier à cette époque là. Au cours de mon année d'internat, les techniciens en radiologie, les technologues de laboratoire, 14000 infirmières et 80000 employés non professionnels avaient tous eu recours à des moyens de pression. Une caricature de Girard dans La Presse montrait un chirurgien qui demandait, à la porte de la salle d'opération : "Quel serait le meilleur moment pour une greffe de rein ? » Un concierge lui répondait « Il faudrait que je consulte l'horaire de la grève. »

Ce matin-là, le $\mathrm{D}^{\mathrm{r}}$ Penfield m'a raconté comment sa carrière avait évolué à son retour d'Angleterre aux États-Unis en 1921. « Lorsque je me on m'a offert $5000 \$$ pour y travailler. Je suis allé parler à l'administrateur de l'hôpital, du nom de Leopold - il avait déjà été secrétaire de Ford — et je lui ai dit que je m'intéressais au problème de la rigidité de décérébration et que je voulais faire un peu de travail en laboratoire. Il a répliqué qu'il y avait déjà quelqu'un de compétent au laboratoire et que si j'avais un problème, il me suffirait de lui en parler. Je lui ai donc répondu "Je ne reste pas". Il a pensé que je voulais négocier et a ajouté "Je vous offre $500 \$$ de plus". » suis présenté à l'Hôpital Henry Ford,
«J'avais alors deux autres offres d'emploi, qui payaient beaucoup moins. J'ai écrit à mon épouse à Devil's Lake, au Wisconsin, pour lui demander si elle accepterait d'aller vivre à Philadelphie ou à New York. Elle m'a répondu par un seul mot : "Tombouctou". »

"L'Hôpital Presbyterian à New York devenait un hôpital à temps plein et j'y ai rencontré le directeur. La proposition avait l'air parfaite : trois mois en neurochirurgie - je n'avais pas fait beaucoup de chirurgie - trois mois en pathologie, trois mois en physiologie, trois mois de congé. J'ai dû lui avouer en toute honnêteté que je n'étais pas suffisamment formé en neurochirurgie. "Mais nous cherchons un neurophysiologiste et un neuropathologiste", a-t-il répondu. Tout est donc tombé en place. Trois ans. » C'est pendant cette période que $\mathrm{D}^{\mathrm{r}}$ Penfield fut invité à réunir toutes les neurosciences sous un même toit pour la première fois au monde en fondant l'Institut neurologique de Montréal.

J'ai mentionné que j'avais lu dans The New York Times une critique de son ouvrage de 1975, The Mystery of the Mind: A Critical Study of Consciousness and the Human Brain. Il m'a répondu « Cette critique était erronée à cause de la remarque selon laquelle $\mathrm{j}$ 'avais fini par admettre le bilinguisme. [Il voulait parler de monothéisme.] »

«Il suffira que quelqu'un trouve comment une forme de matière est transformée en une autre. Comme Einstein l'a dit, tout sera tellement simple. Le cerveau est un système électrique. Il fonctionne avec la même électricité que tout le reste. »

«On découvrira avant longtemps l'interrupteur qui est fermé lorsque je dors la nuit et qui s'ouvre au moment où je me réveille le matin. Bien entendu, lorsqu' on rêve, c'est moitiémoitié. Et l'esprit, lui, il est parti où ? »

«Je pense à toutes ces choses depuis peu de temps seulement, vous savez. Il est difficile de croire à la prédestination et j'en suis venu à conclure qu'il y a un plan et un Dieu qu'il y a un lien entre le Créateur et l'homme créatif. Si l'on regarde l'évolution de l'univers, il faut que ce soit le cas. Il y a des choses qui se produisent lorsqu'on s'y attend le moins. » 
« Vous croyez alors au heureux hasard ?», lui ai-je demandé.

«Non, ce n'est pas ça. », a-t-il répondu. «Vous vous égarez en tenant de tels propos. Si vous faites de votre mieux et que vous travaillez fort, les portes s'ouvriront pour vous. Je le sais; ça a marché dans mon cas. »

Lorsque je me suis levé pour partir, le $\mathrm{D}^{\mathrm{r}}$ Penfield a déclaré « Merci, Alan. Je ne savais pas qu'il y avait de telles personnes. »

Notre conversation a été cordiale et générale. La plupart des arguments qu'il a fait valoir étaient les mêmes qu'il avait manifestement présentés à beaucoup d'autres blancs-becs avant moi. On en trouve d'ailleurs plusieurs dans son autobiographie, No Man Alone: A Neurosurgeon's Life, qu'il avait terminée trois semaines à peine avant notre rencontre.

Le lundi qui a suivi ma visite, un autre interne à qui j'avais raconté ma rencontre m'a informé que Wilder Penfield venait de mourir. "Vous auriez dû venir. Nous sommes entrés dans sa chambre et il est décédé à ce moment-là. Il n'a pas souffert. Vous auriez dû le voir. Il avait l'air songeur lorsqu' il est mort. »

J'ai reçu ce jour-là un appel du directeur de l'Institut neurologique de Montréal, le $\mathrm{D}^{\mathrm{r}}$ William Feindel, qui avait entendu dire que $\mathrm{j}$ 'avais rendu visite au $\mathrm{D}^{\mathrm{r}}$ Penfield et était curieux de savoir de quoi nous avions parlé. En effet, m'a-t-il expliqué, quelques heures après ma rencontre avec lui, $\mathrm{D}^{r}$ Penfield avait sombré dans l'inconscience. Le $\mathrm{D}^{\mathrm{r}}$ Feindel $\mathrm{m}$ 'a informé que j'étais, semble-t-il, la dernière personne à avoir eu une longue conversation avec le $D^{\mathrm{r}}$ Penfield.

Au cours de la même semaine, j'ai assisté au service commémoratif du $\mathrm{D}^{\mathrm{r}}$ Penfield, dans la nef bondée de la
First Presbyterian Church. À mon grand étonnement, j'ai aperçu dans le premier banc de l'église l'une de mes voisines de mon édifice à appartements de la rue Sussex. Nous avions échangé quelques mots dans l'entrée à plusieurs reprises, mais je ne savais pas qu'elle était la petite-fille du Dr Penfield.

Deux ou trois semaines plus tard, Maclean's publiait une entrevue avec le $\mathrm{D}^{\mathrm{r}}$ Penfield : sa dernière, selon le magazine. J'étais toutefois mieux renseigné.

\section{Alan Blum MD \\ Professeur et titulaire de la Chaire dotée en médecine familiale \\ Faculté de médecine de l'Université de \\ l'Alabama \\ Tuscaloosa (Alabama)}

Alan Blum aimerait connaître vos souvenirs du D Penfield : ablum@cchs.ua.edu

JAMC 2011. DOI:10.1503/cmaj.110596 